Table des matières du tome LXXIV, fascicule 2 groups. . . . . $85-98$ L. Monteir 0 , Algèbre du calcul propositionnel trivalent de Heyting . . . 99-109 L. Rudolf, $\theta$-continuous extensions of maps on $\tau X$. . . . . . . . . 111-131 W. Nitka, On convex metric spaces VI . . . . . . . . . . . . . . . 133-144 M. K. Armbrust, An algebraic equivalent of a multiple choice axiom . . 145-140 S. Valenti, Sur la dérivation $k$-pseudo-symétrique des fonctions numériques 147-152 R. Telgársky, Concerning product of paracompact spaces . . . . . . 153-159

Les FUNDAMENTA MATHEMATICAE publient, en langues des congrès internationaux, des travaux consacrés à la Théorie des Ensembles, Topologie, Fondements de Mathématiques, Fonctions Réelles, Algèbre Abstraite.

Chaque volume paraît en 3 fascicules.

Adresse de la Rédaction et de l'Échange:

FUNDAMENTA MATHEMATICAE, Śniadeckich 8, Warszawa 1 (Pologne).

Le prix de ce fascicule est $4.00 \$$.

Tous les volumes sont à obtenir par l'intermédiaire de ARS POLONA-RUCH, Krakowskie Przedmieście 7, Warszawa 1 (Pologne).

DRUKARNIA UNIWERSYTETU JAGIELLONSTIEGO KRAKOWIE

\section{Cardinal properties of lattice ordered groups}

by

\section{J. Jakubík (Košice)}

Pierce [6], [7] defined a cardinal property of complete Boolean algebras as a rule that assigns to any complete Boolean algebra $B$ a cardinal $f B$ such that $f B_{1}=f B_{2}$ whenever $B_{1}$ and $B_{2}$ are isomorphic. He proved that if $f$ is increasing, then each complete Boolean algebra $B$ can be decomposed into a complete direct product of Boolean algebras $B_{i}$ that are homogeneous with regard to $f$. The aim of this note is to prove some analogical results for lattice ordered groups. In $\S 1$ there are studied "increasing" cardinal properties. In $\S 2$ we prove that a complete and laterally complete $l$-group $G$ is a complete direct product of $l$-groups $G_{i}$ such that either any two non-trivial intervals of $G_{i}$ are finite or they have the same length; in $\S 3$ an analogical theorem concerning the powers of intervals is proved.

$\S$ 0. Preliminaries. We shall use the standard notations for lattices and lattice ordered groups (cf. [1], [2]). Let $G$ be an $l$-group; the group operation is denoted by + and the lattice operations by $\wedge, \vee$. If $x, y \in G$ and $x \wedge y=0$, then $x$ and $y$ are said to be disjoint (this fact is denoted by $x \delta y$ ). A subset $X \subset G$ is disjoint if $x>0$ for any $x \in X$ and any two distinct elements of $X$ are disjoint. $Y \delta x(Y \delta X)$ means that the element $x$ (each element of $X$ ) is disjoint with each element $y$ of the set $Y$. Let $G^{+}=\{x \in G: x \geqslant 0\}$ and for any $X \subset G^{+}$write $X^{\delta}=\left\{y \in G^{+}: X \delta y\right\}$. $G$ is laterally complete if for any disjoint subset $\left\{x_{\alpha}\right\} \subset G$ there exists $\bigvee x_{a} \in G$. Let $L$ be a lattice, $a, b \in L, a \leqslant b$. The interval $[a, b]$ is the set $\{x \in L: a \leqslant x \leqslant b\} ;[a, b]$ is a non-trivial interval, if $a \neq b .[a, b]$ is a prime interval when card $[a, b]=2 . L$ is a bounded lattice if it is an interval. A subset $X \subset L$ is convex if $[a, b] \subset X$ whenever $a$ and $b$ belong to $X$. A set $Y \subset L$ is a closed sublattice of $L$ if $\left\{y_{\alpha}\right\} \subset L, \vee y_{\alpha}=y$ implies $y \in Y$, and dually.

Let $I \neq \varnothing$ be a set and for any $i \in I$ let $H_{i}$ be an $l$-group. The complete direct product $H=\Pi^{*} H_{i}(i \in I)$ is the system of all vectors $h=\left(\ldots, h_{i}, \ldots\right)_{i \in I}, h_{i} \in H_{i}$, with operations $+, \wedge, \vee$ that are performed componentwise; then $H$ is an $l$-group. Instead of $h_{i}$ we write also $h(i)$.

7 - Fundamenta Mathematicae, T. LXXIV 
The $l$-subgroup $K$ of $H$ consisting of all elements $k \epsilon H$ such that the set $I(k)=\{i \in I: k(i) \neq 0\}$ is finite is the (discrete) direct product of $l$-groups $H_{i}$. An $l$-subgroup $G$ of $H$ is called a complete subdirect product of $l$-groups $H_{i}$ if for any $i_{0} \in I$ and any $h^{i_{0}} \in H_{i_{0}}$ there is an element $g \in G$ satisfying $g\left(i_{0}\right)=h^{i_{0}}, g(i)=0$ for any $i \in I, i \neq i_{0}$. Let $A, B$ be $l$-ideals of an $l$-group $G$. If $A \cap B=\{0\}, A+B=G$, then $G$ is isomorphic to the direct product of $l$-groups $A$ and $B$; in such a case we write $G=A \times B$. The additive linearly ordered group of all integers (all reals) is denoted by $N\left(R_{0}\right)$.

Let $\beta$ be the class of all bounded lattices containing more than one element and let $\pi$ be the class of all cardinals. Let $f$ be a mapping of the class $B$ into $\pi$ such that $f L_{1}=f L_{2}$ whenever $L_{1}$ is isomorphic to $L_{2}$ and $L_{1}$, $L_{2} \in \Re_{3}$. The mapping $f$ is said to be a cardinal property defined on $\mathfrak{B}_{\text {. }}$ A lattice $L$ is called $f$-homogeneous if $f L_{1}=f L_{2}$ for any two convex sublattices $L_{1}, L_{2}$ of $L$ such that $L_{1}, L_{2} \in \Re$. If card $L=1$, then no sublattice of $L$ belongs to $\mathcal{B}$ and hence $L$ is $f$-homogeneous for any cardinal property $f$. A cardinal property $f$ is increasing if $f L_{1} \leqslant f L_{2}$ for any pair of lattices $L_{1}, L_{2} \in \Re$ such that $L_{1}$ is a convex sublattice of $L_{2}$.

§ 1. Increasing cardinal properties. Let $G \neq\{0\}$ and let $f$ be an increasing cardinal property on the class $\Re$. We shall consider the following conditions on $f$ :

(c) If $t_{i} \in G, 0<t_{i}(i=1,2), f\left[0, t_{1}\right]=f\left[0, t_{2}\right]$ and if $\left[0, t_{1}\right]$ and $\left[0, t_{2}\right]$ are $f$-homogeneous, then $f\left[0, t_{1}+t_{2}\right]=f\left[0, t_{1}\right]$.

$\left(c_{2}\right)$ If $t_{i} \in G, 0<t_{1} \leqslant t_{2} \leqslant \ldots, f\left[0, t_{1}\right]=f\left[0, t_{i}\right], \vee t_{i}=t$ and if the intervals $\left[0, t_{i}\right]$ are $f$-homogeneous $(i=1,2, \ldots)$, then $f[0, t]=f\left[0, t_{1}\right]$ !

Let $A$ be the set of all cardinals $\alpha$ such that $f[a, b]=\alpha$ for some non-trivial interval $[a, b]$ of $G$ and for any $a \in \mathcal{A}$ write

$$
\begin{aligned}
& X_{a}=\{x \in G: x>0, f[0, x] \leqslant \alpha\} \cup\{0\}, \\
& Y_{a}=\{y \in G: y>0, f[0, y]<\alpha\} \cup\{0\}, \\
& Z_{\alpha}=\left(Y_{\alpha}\right)^{\delta}, \quad A_{a}=X_{\alpha} \cap Z_{a} .
\end{aligned}
$$

1.1. Assume that $\left(\mathrm{c}_{1}\right)$ is valid. Let $\alpha \in$ t. Then

(i) the set $A_{\alpha}$ is an ideal of the lattice $G^{+}$and a subsemigroup of $G^{+}$,

(ii) $f[a, b]=\alpha$ for any non-trivial interval $[a, b]$ of $A_{\alpha}$,

(iii) $A_{\alpha} \delta A_{\beta}$ for any $\beta \in \mathcal{A}, \beta \neq \alpha$.

Proof. Let $t \in A_{\alpha}, t>0$. Then $t \in X_{\alpha}$, whence $f[0, t] \leqslant \alpha$. If $f[0, t]<\alpha$, the element $t$ belongs to $Y_{a}$ and since $t \in Z_{a}$, we have $t \delta t$, a contradiction; this implies that $f[0, t]=\alpha$. Let $t_{1} \in A_{\alpha}, 0<t_{1} \leqslant t$. Since $f$ is increasing, $f\left[0, t_{1}\right] \leqslant \alpha$, whence $t_{1} \in X_{\alpha}$. From $Y_{\alpha} \delta t$ if follows that $Y_{\alpha} \delta t_{1}$; thus $t_{1} \in Z_{a}$ and $t_{1} \in A_{\alpha}$. If $0<t_{1}<t$, then the interval $\left[t_{1}, t\right]$ is isomorphic to $\left[0, t-t_{1}\right]$ and $0<t-t_{1}<t$; therefore $f\left[t_{1}, t\right]=\alpha$ and (ii) holds. Let $t_{1}, t_{2} \in A_{a}, t_{1}>0, t_{2}>0$. Clearly, $t_{1} \wedge t_{2} \in A_{a}$, too. According to (ii) and $\left(c_{1}\right)$ $f\left[0, t_{1}+t_{2}\right]=\alpha$ and hence $t_{1}+t_{2} \in X_{a}$. Moreover, from $Y_{a} \delta t_{1}, Y_{a} \delta t_{2}$ we get $Y_{a} \delta\left(t_{1}+t_{2}\right)$; thus $t_{1}+t_{2} \in Z_{\alpha}$ and $t_{1}+t_{2} \in A_{a}$. Since $0<t_{1} \vee t_{2} \leqslant t_{1}+t_{2}$ the element $t_{1} \vee t_{2}$ belongs to $A_{\alpha}$ as well; so the assertion (i) is proved. Let $\beta \in \mathcal{A}, \beta \neq \alpha$. If $A_{\alpha} \delta A_{\beta}$ does not hold, then by (i) we have $t \in A_{\alpha} \cap A_{\beta}$, $t>0$ and according to (ii) $f[0, t]=\alpha, f[0, t]=\beta$, a contradiction.

1.2. If $\left(\mathrm{c}_{1}\right)$ is fulfilled, $t \in G, t>0, f[0, t]=\alpha$, and the interval $[0, t]$ is $f$-homogeneous, then $t \in A_{\alpha}$.

Proof. Clearly $t \in X_{\alpha}$. Let $y \in Y_{a}, t \wedge y=u$. If $u>0$, then $f[0, u]$ $\leqslant f[0, y]<\alpha$ and, at the same time, $[0, u] \subset[0, t]$, whence $f[0, u]=\alpha$, a contradiction. Therefore $Y_{\alpha} \delta t$ and thus $t \in A_{\alpha}$.

From 1.1 and 1.2 we obtain the following:

1.3. Assume that $\left(\mathrm{c}_{1}\right)$ holds and let $\alpha \in \mathcal{A}$. Let $F_{a}$ be the family of all convex sublattices $L_{1}$ of the lattice $G^{+}$such that $0 \in L_{1}$ and $f\left[t_{1}, t_{2}\right]=a$ for any non-trivial interval of $L_{1}$. Then $A_{\alpha}$ is the greatest element of the family $F_{a}$ (ordered by set-inclusion).

1.4. Let $\left(c_{1}\right)$ be valid and for any $\alpha \in \mathcal{A}$ let $B_{a}=\{t \in G$ : there exist $t_{1}, t_{2} \in A_{\alpha}$ such that $\left.-t_{1} \leqslant t \leqslant t_{2}\right\}$. Then (i) $B_{a}$ is an l-ideal of the l-group $G$; (ii) $f[a, b]=\alpha$ for any non-trivial interval $[a, b]$ of $B_{\alpha}$; (iii) $B_{\alpha} \cap B_{\beta}=\{0\}$ for each $\beta \in \mathcal{A}, \beta \neq \alpha$.

Proof. If $t \in B_{a}$, then clearly $-t \epsilon B_{\alpha}$ and from 1.1 (i) it follows that $B_{a}$ is a subsemigroup of $G$; hence $B_{a}$ is a subgroup. of $G$. From this and from the convexity of $A_{\alpha}$ we infer that $B_{\alpha}$ is a convex subset of $G$ and therefore by the definition of $B_{\alpha}$ the element $t \vee 0$ belongs to $B_{\alpha}$ for any $t \in B_{\alpha}$; thus $B_{\alpha}$ is a convex $l$-subgroup of $G$. For proving that $B_{\alpha}$ is normal it suffices to verify that $A_{\alpha}=B_{\alpha}^{+}$is a normal subset of $G$. Let $t \in A_{a}, t>0, x \in G$ and write $t^{\prime}=-x+t+x$. Since the intervals $[0, t]$ and $\left[0, t^{\prime}\right]$ are isomorphic, it follows from 1.1 (ii) and 1.2 that $t^{\prime} \in A_{a}$; thus (i) holds. Let $[a, b]$ be a non-trivial interval of $B_{a}$; then $[a, b]$ is isomorphic to $[0, b-a]$ and $[0, b-a] \subset A_{\alpha}$, whence $f[a, b] \doteq a$. If $x \in B_{\alpha} \cap B_{\beta}, \alpha \neq \beta, x \neq 0$, then $0 \neq|x| \epsilon A_{\alpha} \cap A_{\beta}$, a contradiction.

1.5. Let $\left(\mathrm{c}_{1}\right)$ be valid and let $G_{\alpha}$ be the family of all convex sublattices $L$ of $G$ such that $0 \in L$ and $f\left[t_{1}, t_{2}\right]=\alpha$ for any non-trivial interval of $L$. Then $B_{a}$ is the greatest element of the family $G_{a}$.

Proof. According to 1.4, $B_{\alpha}$ belongs to the family $G_{\alpha}$. Assume that $L \in G_{a}, t \in L, t \neq 0$. If [0, $\left.0 \vee t\right]$ is a non-trivial interval, then it is $f$-homogeneous and $f[0,0 \vee t]=\alpha$, whence by $1.20 \vee t \in A_{\alpha}$. If $[0 \wedge t, 0]$ is a nontrivial interval, then it is $f$-homogeneous and isomorphic to $[0,-(0 \wedge t)]$; thus $-(0 \wedge t) \in A_{\alpha}$. This implies that $t \in B_{\alpha}$ and hence $L \subset B_{\alpha}$.

1.6. THEOREM. Let $f$ be increasing and assume that $\left(\mathrm{c}_{1}\right)$ is valid. For any $\alpha \in \mathcal{H}$ and $g \in G$ let $G_{a}(g)$ be the family of all convex sublattices $L$ of $G$ 
such that $g \in L$ and $f\left[t_{1}, t_{2}\right]=\alpha$ for each non-trivial interval of $L$. Let $G_{a}(g)$ be partially ordered by set-inclusion. Then (i) any family $G_{\alpha}(g)$ contains a greatest element (this will be denoted by $B_{\alpha}(g)$ ); (ii) $B_{a}(0)$ is an l-ideal of $G$ and $B_{\alpha}(g)=B_{\alpha}(0)+g$; (iii) $B_{a}(g) \cap B_{\beta}(g)=\{\dot{g}\}$ for any $\beta \in \mathcal{A}, \beta \neq \alpha$.

Proof. Let $g \in G$. The mapping $\varphi(t)=t+g$ being an automorphism of the lattice $G$, it follows from 1.5 that $B_{\alpha}+g=B_{a}(g)$ is the greatest element of the family $G_{a}(g)$; (ii) and (iii) are consequences of 1.4 .

For any $\alpha \epsilon \mathcal{A}$ let $\bar{A}_{a}$ be the set of all elements $t \in G$ that can be written in the form $t=\vee t_{i},\left\{t_{i}\right\} \subset A_{a}$.

1.7. Let $a \in \mathcal{A}$ and assume that $\left(\mathrm{c}_{1}\right)$ holds. The set $\bar{A}_{\alpha}$ is a closed ideal of the lattice $G^{+}$and a subsemigroup of $G$. If $\beta \in \mathcal{A}, \beta \neq \alpha$, then $\bar{A}_{\alpha} \delta \bar{A}_{\beta}$. $\bar{A}_{\alpha}$ is a normal subset of the group $G$.

Proof. Let $t \in \bar{A}_{a}, t=\bigvee t_{i},\left\{t_{i}\right\} \subset A_{a}, t^{*} \in G, 0 \leqslant t^{*}<t$. Since any lattice ordered group is infinitely distributive ([1]), $t^{*}=t \wedge t^{*}=\bigvee\left(t_{i} \wedge t^{*}\right)$ and $t_{i} \wedge t^{*} \in A_{\alpha}$ by 1.1 ; therefore $t^{*} \in \bar{A}_{\alpha}$. Let $S=\left\{s_{j}\right\}_{j \in J} \subset \bar{A}_{a}, \sup S=s$. For any $s_{j} \in S$ we have $T_{j} \subset A_{\alpha}$ such that $s_{j}=\sup T_{j}$. Thus

$$
s=\sup _{j \in \mathcal{J}}\left(\sup T_{j}\right)=\sup \left(\bigcup_{j \in J} T_{j}\right)
$$

since $\bigcup T_{j} \subset A_{a}$, we have $s \in \bar{A}_{a}$. This proves that $\bar{A}_{a}$ is a closed ideal of the lattice $G^{+}$. Let $t=\bigvee_{i \in I} t_{i}, t^{\prime}=\bigvee_{j \in J} t_{j}^{\prime},\left\{t_{i}\right\},\left\{t_{j}^{\prime}\right\} \subset A_{a}$. Then $t+t^{\prime}$ $=\bigvee_{i \in I} \bigvee_{j \in J}\left(t_{i}+t_{j}^{\prime}\right)$, whence $t+t^{\prime} \in \bar{A}_{\alpha}$. Further, let $\beta \in \mathcal{A}, \beta \neq \alpha, t=\bigvee t_{i}$, $\left\{t_{i}\right\} \subset A_{a}, t^{\prime}=\dot{V} t_{j}^{\prime},\left\{t_{j}^{\prime}\right\} \subset A_{\beta}$. According to 1.1, $t_{i} \wedge t_{j}^{\prime}=0$, and thus, by using infinite distributivity, $t \wedge t^{\prime}=0$. From the normality of $A_{a}$ it follows that $\bar{A}_{a}$ is also normal.

Let us put $\bar{B}_{\alpha}=\left\{t \in G\right.$ : there exist elements $t_{1}, t_{2} \in \bar{A}_{\alpha}$ such that $\left.-t_{1} \leqslant t \leqslant t_{2}\right\}$.

From 1.7 we immediately obtain the following:

1.7.1. Let $\alpha \in \mathcal{A}$ and assume that $\left(\mathrm{c}_{1}\right)$ is fulfilled. The set $\bar{B}_{\alpha}$ is an $l$-ideal of $G$. If $\beta \in \mathcal{A}, \beta \neq \alpha$, then $\bar{B}_{\alpha} \cap \bar{B}_{\beta}=\{0\}$.

Let us now assume that $G$ is a complete $l$-group (i.e., that the lattice $G$ is relatively complete), $g \in G^{+}$and for any $a \in \mathcal{A}$ write

$$
g_{a}=\sup \left\{t \in A_{a}: t \leqslant g\right\} .
$$

By the definition of $\bar{A}_{\alpha}, g_{\alpha} \in \bar{A}_{\alpha}$.

1.8. Let $G$ be a complete l-group and suppose that $\left(\mathrm{c}_{1}\right)$ holds. Then $g=\bigvee g_{a}(\alpha \in \mathcal{A})$ for any $g \in G^{+}$. If $g=\bigvee h_{\alpha}(\alpha \in \mathcal{A}), h_{\alpha} \in \bar{A}_{\alpha}$ for each $\alpha \in \mathcal{A}$, then $h_{\alpha}=g_{\alpha}$.

Proof. Put $\bigvee g_{a}=h$. Clearly $h \leqslant g$. Assume that $h<g$ and write $-h+g=k$; further, let

(1)

$$
\beta=\min \{f[0, b]: 0<b \leqslant k\} .
$$

There exists $b_{0} \in G, 0<b_{0} \leqslant k$ such that $f\left[0, b_{0}\right]=\beta$. Then for any $b_{1}>0, b_{1} \leqslant b_{0}$ we have $f\left[0, b_{1}\right] \leqslant f\left[0, b_{0}\right]$ and according to (1) $f\left[0, b_{1}\right] \geqslant \beta$, whence the interval $\left[0, b_{0}\right]$ is $f$-homogeneous. Thus, by $1.2, b_{0} \in A_{\beta}$. There is a subset $\left\{t_{i}\right\} \subset A_{\beta}$ such that $g_{\beta}=\bigvee t_{i}$. Therefore, we have

$$
g_{\beta}+b_{0}=\left(\bigvee t_{i}\right)+b_{0}=\bigvee\left(t_{i}+b_{0}\right)
$$

and $t_{i}+b_{0} \in A_{\beta}$ by 1.1. Moreover, $t_{i}+b_{0} \leqslant g_{\beta}+b_{0} \leqslant h+k=g$, whence (by the definition of $\left.g_{\beta}\right) \vee\left(t_{i}+b_{0}\right) \leqslant g_{\beta}$; thus $g_{\beta}+b_{0} \leqslant g_{\beta}$, a contradiction. Therefore $\bigvee g_{\alpha}=g$. If $\bigvee h_{\alpha}=g, h_{\alpha} \in \bar{A}_{\alpha}$, then for any $\alpha_{0} \in t$

$$
g_{a_{0}}=g_{\alpha_{0}} \wedge g=\bigvee\left(g_{a_{0}} \wedge h_{a}\right)=g_{\alpha_{0}} \wedge h_{\alpha_{0}}
$$

by 1.4. Analogously, we obtain $h_{\alpha_{0}}=g_{\alpha_{0}} \wedge h_{\alpha_{0}}$, whence $g_{\alpha_{0}}=h_{\alpha_{0}}$.

In 1.9-1.20 we assume that $G$ is a complete $l$-group and that $\left(c_{1}\right)$ is valid.

1.9. For any $\alpha \in \mathcal{A}$ and any $g \in G^{+}$, let $\varphi_{\alpha}(g)=g_{\alpha}$. Then $\varphi_{\alpha}$ is a homomorphism of the lattice ordered semigroup $G^{+}$onto the lattice ordered semigroup $\bar{A}_{\alpha}$. For $g \in \bar{A}_{\alpha}$ we have $\varphi_{\alpha}(g)=g$ and $\varphi_{\beta}(g)=0$ whenever $\beta \in \mathfrak{A}$, $\beta \neq \alpha$.

Proof. Let $g, h \in G^{+}$. Then $g_{\alpha_{1}} \delta g_{a_{2}}$ for any $\alpha_{1}, \alpha_{2} \in \mathcal{A}, \alpha_{1} \neq a_{2}$, and thus, by using infinite distributivity, $g \wedge h=\bigvee\left(g_{a} \wedge h_{a}\right)$; further, we have $g \vee h=\vee\left(g_{a} \vee h_{a}\right)$. Since by $1.7 g_{a} \wedge h_{a}$ and $g_{a} \vee h_{\alpha}$ belong to $\bar{A}_{\alpha}$, it follows from 1.8 that $(g \wedge h)_{\alpha}=g_{\alpha} \wedge h_{\alpha},(g \vee h)_{\alpha}=g_{a} \vee h_{\alpha}$. Moreover,

$$
g+h=\bigvee_{\alpha \in \mathcal{A}} g_{a}+h=\bigvee_{\alpha \in \mathcal{A}}\left(g_{a}+h\right)=\bigvee_{\alpha \in \mathcal{A} \in} \bigvee_{\beta \in \mathcal{A}}\left(g_{a}+h_{\beta}\right)
$$

If $\alpha \neq \beta$, then $g_{\alpha} \delta h_{\beta}$, whence (ef. [1]) $g_{\alpha}+h_{\beta}=g_{a} \vee h_{\beta} \leqslant\left(g_{a}+h_{\alpha}\right) \vee\left(g_{\beta}+h_{\beta}\right)$; thus $g+h=\bigvee_{\alpha \in \mathcal{A}}\left(g_{\alpha}+h_{\alpha}\right)$; therefore, according to 1.7 and $1.8,(g+h)_{\alpha}$ $=g_{a}+h_{a}$. Hence $\varphi_{a}$ is an homomorphism. From the definition of $g_{\alpha}$ it follows immediately that for $g \in \bar{A}_{\alpha}$ we have $g_{\alpha}=g$; moreover, from $\bar{A}_{\alpha} \delta \bar{A}_{\beta}$ we obtain $g_{\beta}=0$ for any $\beta \in \mathfrak{A}, \beta \neq \alpha$.

1.10. Let $g, h, g^{\prime}, h^{\prime} \in G^{+}, g-h=g^{\prime}-h^{\prime}, \alpha \in \mathcal{A}$. Then $g_{\alpha}-h_{\alpha}=g_{\alpha}^{\prime}-h_{\alpha}^{\prime}$.

Proof. Since $G$ is a complete $l$-group, $G$ is commutative. Hence $g+h^{\prime}=g^{\prime}+h$ and thus, by $1.9, g_{\alpha}+h_{\alpha}^{\prime}=g_{\alpha}^{\prime}+h_{\alpha}$.

For any $k \in G$ there exist elements $g, h \in G^{+}$such that $k=g-h$; put $k_{\alpha}=g_{\alpha}-h_{\alpha}$. According to $1.10 k_{\alpha}$ is uniquely determined by $k$. From 1.9 and 1.10 we obtain the following:

1.11. If $g \in \bar{B}_{a}, \beta \in A, \beta \neq \alpha$, then $g_{a}=g, g_{\beta}=0$. The mapping $g \rightarrow g_{a}$ is a homomorphism of the group $G$ onto the group $\bar{B}_{\alpha}$.

1.12. The mapping $g \rightarrow g_{\alpha}$ is a homomorphism of the lattice $G$ onto the lattice $\bar{B}_{a}$. 
Proof. Let $g, h \in G$. There exists $k \in G$ such that $k \leqslant g, k \leqslant h$. Then $g-k, h-k \in G^{+}$and thus, according to 1.9 , and 1.11

$$
\begin{aligned}
(g \vee h)_{\alpha}-k_{\alpha} & =[(g \vee h)-k]_{\alpha}=[(g-k) \vee(h-k)]_{\alpha} \\
& =(g-k)_{\alpha} \vee(h-k)_{\alpha}=\left(g_{\alpha} \vee h_{\alpha}\right)-k_{a} ;
\end{aligned}
$$

therefore $(g \vee h)_{i=}=g_{a} \vee h_{\alpha}$. The proof for the operation $\wedge$ is analogous.

Write $H=\Pi^{*} \bar{B}_{\alpha}(\alpha \in \mathcal{A})$ and consider the mapping $\varphi: G \rightarrow H$ defined by $\varphi(g)=\left(\ldots, g_{\alpha}, \ldots\right)$. Let $\varphi(G)=H_{0}$.

1.13. The mapping $\varphi$ is an isomorphism of $G$ onto $H_{0}$.

Proof. According to 1.11 and 1.12, $\varphi$ is a homomorphism, whence it suffices to prove that from $\varphi\left(g_{1}\right)=\varphi\left(g_{2}\right)$ follows $g_{1}=g_{2}$. Let $\varphi\left(g_{1}\right)$ $=\varphi\left(g_{2}\right)$ and write $g=g_{1} \vee g_{2}-g_{1} \wedge g_{2}$. Then $\varphi(g)=0$, whenee $g_{a}=0$ for each $\alpha \epsilon \mathfrak{t}$; moreover, $g \in G^{+}$, whence by $1.8 \mathrm{~g}=\bigvee g_{\alpha}=0$ and thus $g_{1}=g_{2}$.

According to 1.11, for any $\alpha_{0} \in \mathfrak{A}$ and any $g^{\alpha_{0}} \in \bar{B}_{\alpha_{0}}$ there is an element $h \in H^{0}$ (namely $\left.h=\varphi\left(g^{\alpha_{0}}\right)\right)$ such that $h\left(\alpha_{0}\right)=g^{\alpha_{0}}, h(\alpha)=0$ for any $\alpha \in \mathcal{A}$, $\alpha \neq \alpha_{0}$. Thus $H_{0}$ is a complete subdirect product of $l$-groups $\bar{B}_{\alpha}(\alpha \in \mathcal{H})$.

1.14. Let $P_{i}(i \in I)$ be l-groups, $P=\Pi^{*} P_{i}(i \in I)$. Assume that an $l$-subgroup $Q$ of $P$ is a complete subdirect product of l-groups $P_{i}$ and that $Q$ is laterally complete. Then $Q=P$.

Proof. Let $p=\left(\ldots, p_{i}, \ldots\right) \in P$. To any $p_{i}$ there correspond elements $u_{i}, v_{i} \in P_{i}^{+}$such that $p_{i}=u_{i}-v_{i}$. Write $u=\left(\ldots, u_{i}, \ldots\right), v=\left(\ldots, v_{i}, \ldots\right)$. Since $Q$ is a complete subdirect product of $l$-groups $P_{i}$, for any $i \in I$ there are elements $u^{i}, v^{i} \in Q$ such that $u^{i}(i)=u_{i}, v^{i}(i)=v_{i}, u^{i}(j)=v^{i}(j)$ $=0$ whenever $j \epsilon I, j \neq i$. The system $\left\{u^{i}: i \in I\right\}$ is disjoint, whence $u=\bigvee u^{i} \in Q$; similarly, $v=\bigvee v^{i} \in Q$, whence $p \in Q$ and thus $P=Q$.

By summarizing, we have the following assertion:

1.15. THEOREM. Let $G$ be a complete l-group. Assume that $f$ is increasing and that $\left(\mathrm{c}_{1}\right)$ is fulfilled. For any $\alpha \in \mathcal{A}$ let $\bar{B}_{\alpha}$ be the system of all $b \in G$ such that there are subsets $\left\{t_{i}\right\},\left\{t_{j}^{\prime}\right\} \subset A_{a}$ satisfying $-\left(\bigvee t_{i}\right) \leqslant b \leqslant \bigvee t_{j}^{\prime}$. Then $\bar{B}_{a}$ are convex l-subgroups of $G$ and $G$ is isomorphic to a complete subdirect product of l-groups $\bar{B}_{\alpha}(\alpha \in \mathcal{A})$. If $G$ is laterally complete, $G$ is isomorphic to a complete direct product of l-groups $\bar{B}_{a}(\alpha \in A)$.

The lattices $\bar{B}_{a}$ need not, in general, be $f$-homogeneous, and this is the reason for searching for a "better" complete subdirect decomposition of $G$. (Example. For any non-trivial interval $[a, b] \subset G$ put $f[a, b]$ $=\max \left\{\operatorname{card}[a, b], \mathrm{s}_{0}\right\}$. Then $f$ is increasing and satisfies $\left(c_{1}\right)$ (cf. 3.1) Let $I$ be an infinite set, $C_{i}=E$ for each $i \in I, G=\Pi^{*} C_{i}(i \in I)$ and let $\alpha=s_{0}$. Denote by $H$ the diserete direct product of $l$-groups $C_{i}(i \in I)$ We have $A_{a}=H^{+}, B_{a}=H$. Since each element $0<g \in G$ is the supremum of some subset of $H^{+}$, we get $\bar{B}_{\alpha}=G$. Let $g \in G, g(i)=1$ for each $i \in I$. Clearly, $f[0, g]>s_{0}$ and $f[0, h]=s_{0}$ for any $\theta<h \in H$. Therefore $\bar{B}_{\alpha}$ is not $f$-homogeneous.) Under the same assumptions as in 1.15 let $\alpha \in \mathcal{A}$ be fixed, $\bar{B}_{a} \neq\{0\}$. Choose any maximal disjoint subset $\left\{a_{i}\right\}_{i \in I}$ of the $l$-group $\bar{B}_{\alpha}$. Hence $a_{i} \in A_{\alpha}$ for each $i \in I$. Let $b \in \bar{B}_{\alpha}, b>0$. Then there is a subset $\left\{t_{j}\right\} \subset A_{a}, t_{j}>0, \bigvee t_{j}=b$. For any $t_{j}$ there exists an $a_{i}$ such that $t_{j} \wedge a_{i}>0$; thus $b \wedge a_{i}>0$, and therefore $\left\{a_{i}\right\}_{i \in I}$ is a maximal disjoint subset of the $l$-group $\bar{B}_{a}$. For any $i \in I$ write $C_{i}=\left\{b \in \bar{B}_{a}:|b| \wedge a_{j}=0\right.$ for each $j \in I, j \neq i\}$. It is known that $C_{i}$ is a closed convex $l$-subgroup of $\bar{B}_{a}$ (cf. [2], p. 119, Proposition 12).

1.16. $C_{i} \cap C_{j}=\{0\}$ for any $i, j \in I, i \neq j$.

Proof. Let $x \in C_{i} \cap C_{j}, i \neq j$. Then $|x| \epsilon C_{i}$, whence $|x| \delta a_{k}$ for any $k \in I, k \neq i$; moreover, from $x \in C_{j}$ we obtain $|x| \delta a_{i}$. If $x \neq 0$, then $|x|$ $\notin\left\{a_{m}\right\}_{m \in I}$ and $\left\{a_{m}\right\}_{m \in I} \cup\{|x|\}$ is a disjoint set, a contradiction.

For any $0 \leqslant g \in \bar{B}_{a}$ and any $i \in I$ write $g_{i}=\sup \left\{t \in C_{i}: t \leqslant g\right\}$. Since $C_{i}$ is a closed sublattice of $\bar{B}_{a}$ and $\bar{B}_{a}$ is a closed sublattice of $G$, we have $g_{i} \in C_{i}$.

1.17. $g=\bigvee g_{i}$ for any $g \in \bar{B}_{\alpha}, g \geqslant 0$.

Proof. Clearly, $g_{i} \leqslant g$ for each $g_{i}$; let $\vee g_{i}=h$ and assume $h<g$; let $g-h=k$. Then there exists an $i_{0} \in I$ such that $k \wedge a_{i_{0}}=a>0$. Thus $a \in C_{i_{0}}, g_{i_{0}}+a \in C_{i_{0}}$ and $g_{i_{0}}<g_{i_{0}}+a \leqslant h+k=g$; hence $g_{i_{0}}$ is not the greatest element of the set $\left\{t \in C_{i_{0}}: t \leqslant g\right\}$, which is a contradiction.

Now the same method that was used in 1.9-1.15 yields (by applying 1.16 and 1.17 ) the following:

1.18. The $l$-group $\bar{B}_{\alpha}$ is isomorphic to a complete subdirect product of l-groups $C_{i}(i \in I)$.

An element $e$ of an $l$-group $H$ is called a weak unit of $H$ if $h \wedge e>0$ whenever $h \in H, h>0$.

1.19. Let $e$ be a weak unit of a complete l-group $H, h \in H, h \geqslant 0$. Then

$$
\bigvee_{n=1}^{\infty}(n e \wedge h)=h
$$

This assertion is proved in [8], p. 97, for the case where $H$ is a complete vector lattice (" $K$-space"), but the proof remains valid also for complete $l$-groups.

Let us remark that for any $i \in I$ the element $a_{i}$ is a weak unit of $A_{i}$ (otherwise there would exist a positive element $d \epsilon A_{i}$ such that $a_{i} \delta d$ and then, according to 1.16 , we would have $a_{j} \delta d$ for each $j \in I$, whence $\left\{a_{i}\right\}_{i \in I} \cup\{d\}$ would be a disjoint set, a contradiction).

1.20. If $\left(c_{2}\right)$ holds, then $f[a, b]=\alpha$ for any non-trivial interval $[a, b]$ of $C_{i}$.

Proof. Since $[a, b]$ is isomorphic to $[0, b-a]$, it suffices to prove that $f[0, t]=\alpha$ for any $t \epsilon C_{i}, t>0$. From $a_{i} \in A_{\alpha}$ it follows that $n a_{i} \in A_{n}$ for any positive integer $n$, and since $a_{i}$ is a weak unit of $\bar{B}_{a}, 0<n a_{i} \wedge t \in A_{a}$, 
we have $f\left[0, n a_{i} \wedge t\right]=\alpha$ and all these intervals are $f$-homogeneous. By 1.19

$$
\bigvee_{n=1}^{\infty}\left(n a_{i} \wedge t\right)=t
$$

and thus according to $\left(\mathrm{c}_{2}\right) f[0, t]=\alpha$.

From 1.15, 1.18 and 1.20 we obtain:

1.21. THEOREM. Let $G$ be a complete l-group and let $f$ be an increasing cardinal property satisfying $\left(\mathrm{c}_{1}\right)$ and $\left(\mathrm{c}_{2}\right)$. Then $G$ is isomorphic to a complete subdirect product of $f$-homogeneous l-groups. If $G$ is also laterally complete, then it is isomorphic to a complete direct product of $f$-homogeneous 7 -groups.

Under the same assumptions as in Theorem 1.21 let $\alpha \in \mathcal{A}$ be fixed, $\bar{B}_{\alpha} \neq\{0\}$ and let $A_{0}=\left\{a_{i}\right\}_{i \in I_{n}}$ be the system of all atoms of the lattice $\bar{B}_{\alpha}^{+}$. There exists a maximal disjoint subset $A_{0}=\left\{a_{i}\right\}_{i \in I}$ such that $I_{0} \subset I$. Let $i_{0} \in I_{0}$. Since [0, $\left.a_{i_{0}}\right]$ is a prime interval, it is a chain and thus (cf. [5], Thm. $\left.1^{\prime}\right)$ there exists a direct decomposition

$$
\bar{B}_{\alpha}=R_{i_{0}} \times Q_{i_{0}}
$$

such that $R_{i_{0}}$ is linearly ordered and $a_{i_{0}} \in R_{i_{0}}$. Moreover, $R_{i_{0}}$ is complete. and $a_{i_{0}}$ is an atom of $R_{i_{0}}^{+}$, whence $R_{i_{0}}$ is isomorphic to the $l$-group $N$ consisting of all integers (cf. [1]). Obviously $A^{\prime}=A_{0} \backslash\left\{a_{i_{0}}\right\}$ is a subset of $Q_{i_{0}}$ and $A^{\prime}$ is a maximal disjoint subset of $Q_{i_{0}}$; therefore, $R_{i_{0}}=C_{i_{0}}$. Now let $i \in I I_{0}$ and assume that $C_{i}$ contains a prime interval $[u, v]$. Then $v-u=a_{i_{0}}$ is an atom of the lattice $\bar{B}_{a}^{+}, a_{i_{0}} \in O_{i_{0}} \cap C_{i}$; according to 1.16 , $C_{i_{0}} \cap C_{i}=\{0\}$, a contradiction. Thus for $i \in I \backslash I_{0}$ each non-trivial interval of the $l$-group $C_{i}$ is infinite. Hence from 1.21 follows:

1.22. THeonem. Let $G \neq\{0\}$ be a complete l-group and let $f$ be an increasing cardinal property satisfying $\left(\mathrm{c}_{1}\right)$ and $\left(\mathrm{c}_{2}\right)$. Then there exists a complete subdirect decomposition of $G$ with factors $C_{k}(k \in K)$ such that (i) each factor $C_{k}$ is $f$-homogeneous and (ii) for any $k \in K$ either $C_{k}$ is isomorphic to $N$ or each non-trivial interval of $C_{k}$ is infinite.

1.23. According to the constructions of subdirect decompositions of $G$ (Thm. 1.15) and of $B_{a}$ (ef. 1.17 and 1.18), we may assume that the factors $C_{k}$ in 1.22 are $l$-ideals of $G$ and that, for any $g \in G^{+}, g=V g^{k}(k \in K)$, where $g^{k}$ is the $k$-th component of $g$ with regard to the subdirect decomposition of $G$
described in 1.22 .

$\$ 2$. Lengths of intervals of a lattice ordered group. Let $[a, b]$ be a nontrivial interval of a lattice $L$ and let $R[a, b]$ be the system of all maximal chains of the interval $[a, b]$. We define the length $s[a, b]$ of $[a, b]$ by

Write $f_{1}[a, b]=\max \left\{s[a, b], \mathrm{N}_{0}\right\}$.
2.1. Let $L$ be a complete infinitely distributive lattice, $R \in \Re L$. Let 0 be the least element of $L, a \in L, a>0$. Then $R_{1}=\{r \wedge a: r \in R\}$ belongs to $R[0, a]$.

Pro of. Clearly $R_{1}$ is a chain, $R_{1} \subset[0, a]$; assume that $R_{1} \notin R[0, a]$. Then there exists $b \in[0, a] \backslash R_{1}$ such that $R_{1} \cup\{b\}$ is a chain. Let $R_{u}\left(R_{v}\right)$ be the set of all $r \in R$ such that $r \wedge a<b \quad(r \wedge a>b)$. Since $L$ is complete, there exists an $r_{0} \in R$ such that $r_{0}=\wedge r_{i}\left(r_{i} \in R_{v}\right)$. Then $r_{0} \wedge a=\wedge\left(r_{i} \wedge a\right)$ $>b \quad\left(r_{0}^{*} \wedge a=b\right.$ cannot hold, since $\left.r_{0} \wedge a \in R_{1}, b \notin R_{1}\right)$. Write $r_{1}=\vee r$ $\left(r_{j} \in R_{u}\right)$. Clearly, $r_{0} \geqslant r_{1}$; if $r_{0}=r_{1}$, then $r_{0} \wedge a=\bigvee\left(r_{j} \wedge a\right) \leqslant b$, a contradiction. If $r_{0}>r_{1}$, then $\left[r_{1}, r_{0}\right]$ is a prime interval, whence the set $L_{1}=\left\{r_{0}, r_{1}\right.$, $\left.a \wedge r_{0}, b, a \wedge r_{1}\right\}$ is a non-modular sublattice of $L$; a contradiction.

The assertion dual to 2.1 can be proved similariy.

2.2. Let $L$ be a complete infinitely distributive lattice, $[u, v] \subset L, u<v$, $R \in \mathcal{R} L$. Then there is an $R_{1} \in \mathcal{R}[u, v]$ such that card $R_{1} \leqslant \operatorname{card} R$.

Proof. Let 0 be the least element of $L$. According to $2.1 R^{\prime}=\{r \wedge v$ : $r \in R\}$ belongs to $R[0, v]$ and hence, by the assertion dual to $2.1, R_{1}$ $=\left\{r^{\prime} \vee u: r^{\prime} \in R^{\prime}\right\} \in \mathcal{R}[u, v]$. Obviously $\operatorname{card} R_{1} \leqslant \operatorname{card} R^{\prime} \leqslant \operatorname{card} R$.

Let $G$ be a complete $l$-group. Since $G$ is infinitely distributive, it follows from 2.2 that $f_{\mathrm{i}}$ is increasing.

- 2.3. Let $G$ be a complete l-group. Then $f_{1}$ satisfies $\left(c_{1}\right)$.

Proof. Let $t_{i} \in G, t_{i}>0(i=1,2), f_{1}\left[0, t_{1}\right]=f_{2}\left[0, t_{2}\right]=\alpha, f_{1}\left[0, t_{1}+\right.$ $\left.+t_{2}\right]=\beta$. Since $f_{1}$ is increasing, $a \leqslant \beta$. The lattices $\left[0, t_{2}\right]$ and $\left[t_{1}, t_{1}+t_{2}\right]$ are isomorphic, and thus $f_{1}\left[t_{1}, t_{1}+t_{2}\right]=\alpha$. There are chains $R_{1} \in \mathcal{R}\left[0, t_{1}\right]$, $R_{2} \in R\left[t_{1}, t_{1}+t_{2}\right]$ such that $\operatorname{card} R_{1} \leqslant \alpha$, card $R_{2} \leqslant \alpha$; the set $R_{1} \cup R_{2}$ belongs to $\mathcal{R}\left[0, t_{1}+t_{2}\right]$ and $\operatorname{card} R \leqslant \alpha$; hence $\beta=\alpha$.

Let $\mathfrak{A}_{1}=\left\{f_{1}[a, b]:[a, b] \subset G, a<b\right\}$. From 2.3 and 1.6 follows:

2.4. THeorem. Let $G \neq\{0\}$ be a complete l-group. Let $a \epsilon \mathfrak{A}_{1}, a>s_{0}$ $\left(\alpha=s_{0}\right)$. For any $g \in G$ let $G_{a}^{1}(g)$ be the family of all convex sublattices $L$ of $G$ such that $g \in L$ and the length of each non-trivial interval of $L$ equals $\alpha$ (equals or is less than $\alpha$ ). Then (i) any family $G_{a}^{1}(g)$ has a greatest element $B_{a}(g)$, (ii) $B_{\alpha}(0)$ is an $l$-ideal of $G$ and $B_{\alpha}(g)=B_{\alpha}(0)+g$.

Let us remark that for a non-complete $l$-group $G f_{1}$ need not be increasing. Example: Let $A(B)$ be the additive group of all rational (real) numbers with the natural order, $G=A \times B, t_{0}=(0,0), t_{1}=(0,1)$, $t_{2}=(1,1)$. Then $f_{1}\left[t_{0}, t_{1}\right]=c$ (the power of the continuum). Let $R$ $=\{(r, r): 0 \leqslant r \leqslant 1, r \in A\} . R$ is a maximal chain of the lattice $\left[t_{0}, t_{2}\right]$ and $\operatorname{card} R=\mathrm{s}_{0}$; hence $f_{1}\left[t_{0}, t_{2}\right]=\mathrm{s}_{0}$.

2.5. Let $G$ be a complete l-group. Then $f_{1}$ fulfils $\left(c_{2}\right)$.

Proof. Let $0<t_{i} \in G, f_{1}\left[0, t_{i}\right]=\alpha$ and let $\left[0, t_{i}\right]$ be $f_{1}$-homogeneous $(i=1,2, \ldots) ; t_{1} \leqslant t_{2} \leqslant \ldots, \forall t_{i}=t$. Since $f_{1}$ is increasing, $f_{1}[0, t] \geqslant \alpha$. Let $S$ be the system of all intervals $\left[t_{i}, t_{i+1}\right]\left(t_{0}=0, i=1,2, \ldots\right)$ that 
are non-trivial. According to the $f_{1}$-homogenity of $\left[0, t_{i+1}\right], f_{1}\left[t_{i}, t_{i+1}\right]=0$ whenever $\left[t_{i}, t_{i+1}\right]$ is non-trivial. For each $L_{i} \in S$ there exists a maxima chain $R_{i} \in R L_{i}$ such that card $R_{i} \leqslant \alpha$; let $R$ be the union of all these $R_{i}$. Then $R \in \mathcal{R}[0, t]$ and card $R \leqslant \alpha$, whence $f_{1}[0, t]=\alpha$.

From 2.3, 2.5 and 1.22 we obtain:

2.6. THEOREM. Let $G$ be a complete $l$-group. Then $G$ is isomorphic to a complete subdirect product of $l$-groups $C_{k}(k \in K)$ such that for each $k \in K$ either (i) every interval of $C_{k}$ is finite, or (ii) any two non-trivia intervals of $C_{k}$ have the same length $\alpha_{k} \geqslant \mathrm{~s}_{0}$. If $G$ is laterally complete, then $G$ is isomorphic to a complete direct product of l-groups $C_{k}$.

Now we may ask whether we could obtain analogical results if we define the length of a bounded lattice $L(\operatorname{card} L>1)$ by the rule

$$
s^{\prime} L=\sup \{\operatorname{card} R: R \in R L\} .
$$

Put $f_{2} L=\max \left\{s^{\prime} L, s_{0}\right\}$. Clearly, $f_{2}$ is increasing. Let $G \neq\{0\}$ be an $l$-group, $\mathfrak{A}_{2}=\left\{f_{2}[a, b]:[a, b] \subset G, a<b\right\}$.

2.7. $f_{2}$ fulfils $\left(\mathrm{c}_{1}\right)$.

Proof. Let $0<t_{i} \in G, f_{2}\left[0, t_{i}\right]=\alpha(i=1,2)$. Then $f_{2}\left[t_{1}, t_{1}+t_{2}\right]=\alpha$ and, since $f_{2}$ is increasing, $f_{2}\left[0, t_{1}+t_{2}\right] \geqslant \alpha$. Let $R \in \mathcal{R}\left[0, t_{1}+t_{2}\right]$ and write $R_{1}=\left\{r_{1}: r_{1}=r \wedge t_{1}, r \in R\right\}, R_{2}=\left\{r_{2}: r_{2}=r \vee t_{1}, r \in R\right\}$. The set $R_{1}\left(R_{2}\right)$ is a chain in $\left[0, t_{1}\right]\left(\left[t_{1}, t_{1}+t_{2}\right]\right)$, whence card $R_{1} \leqslant \alpha$, card $R_{2} \leqslant \alpha$. Since $G$ is distributive and $r$ is the relative complement of the element $t_{1}$ in the interval $\left[r_{1}, r_{2}\right]$, the pair of elements $\left(r_{1}, r_{2}\right)$ uniquely determines $r$. Thus card $R \leqslant \operatorname{card}\left(R_{1} \times R_{2}\right) \leqslant \alpha$. This proves that $f_{2}\left[0, t_{1}+t_{2}\right]=\alpha$.

From 2.7 and 1.6 we obtain the following:

2.8. Let $G \neq\{0\}$ be an l-group, $\alpha \in \mathfrak{A}_{2}, \alpha>s_{0}\left(\alpha=s_{0}\right)$. For any $g \in G$ let $G_{a}^{\prime}(g)$ be the family of all convex sublattices $L$ of $G$ such that $g \in L$ and for any non-trivial interval $L_{1}$ of $L s^{\prime} L_{1}=\alpha\left(s^{\prime} L_{1} \leqslant s_{0}\right)$. Then (i) each family $G_{a}^{\prime}(g)$ has a greatest element $B_{\alpha}^{\prime}(g)$, (ii) $B_{\alpha}^{\prime}(0)$ is an $l$-ideal of $G$ and $B_{\alpha}^{\prime}(g)$ $=B_{\mathrm{a}}^{\prime}(0)+g$.

There exist complete $l$-groups $G$ such that $f_{2}$ fails to satisfy $\left(c_{2}\right)$. Example: Let $I=\{1,2, \ldots\}, G_{i}=N$ for each $i \in I, G=\Pi^{*} G_{i}$. For any $i \in I$ define $t_{i}$ by

$$
t_{i}(j)=1 \text { for } j \in I, j \leqslant i \text {, and } t_{i}(j)=0 \text { otherwise. }
$$

Further, let $\overline{0}, \overline{1} \in G$ such that $\overline{0}(j)=0, \overline{1}(j)=1$ for each $j \in I$. Clearly, $s^{\prime}\left[\overline{0}, t_{i}\right]=i+1$, whence $f_{\bar{z}}\left[\overline{0}, t_{i}\right]=s_{0}$ and all intervals $\left[\overline{0}, t_{i}\right]$ are $f_{2}$-homo geneous. We have $\overline{0}<t_{1}<t_{2}<\ldots, V t_{i}=\overline{1}$ and the interval. $[\overline{0}, \overline{1}]$ is isomorphic to the Boolean algebra $B$ consisting of all subsets of the set $I$. There is a chain $R$ in $B$ such that card $R=c$ (cf. [4]). Thus $f_{2}[\overline{0}, \overline{1}] \neq \mathrm{s}_{0}$. Let $L$ be a lattice, $L_{1} \subset L$. The set $L_{1}$ is dense in $L$, if $L_{1} \frown[a, b] \neq \emptyset$ for any non-trivial interval $[a, b] \subset L$. He define the reduced length $s^{*} L$ of a bounded lattice by $s^{*} L=\min \{\alpha \in \pi$ : there exists an $R \in R L$ and a dense subset $L_{1}$ of $R$ such that card $\left.L_{1}=\alpha\right\}$. By the same method as in 2.1-2.6 analogical results for the reduced length can be proved.

$\S 3$. The powers of intervals of an l-group. Let $G$ be an l-group, $G \neq\{0\}$. For any non-trivial interval $[a, b] \subset G$ we write $f_{3}[a, b]=\max \{\operatorname{card}[a, b]$, $\left.s_{0}\right\}$. Obviously, $f_{3}$ is increasing.

3.1. $f_{3}$ satisfies $\left(\mathrm{c}_{1}\right)$.

Pro of. Let $0<t_{i} \in G, f_{3}\left[0, t_{i}\right]=\alpha(i=1,2), f_{3}\left[0, t_{1}+t_{2}\right]=\beta$. Then $f_{3}\left[t_{1}, t_{1}+t_{2}\right]=\alpha \leqslant \beta$ and each element $t \in\left[0, t_{1}+t_{2}\right]$ is uniquely determined by the pair $\left(t \wedge t_{1}, t \vee t_{1}\right)$. Since $t \wedge t_{1} \in\left[0, t_{1}\right], t \vee t_{1} \in\left[t_{1}, t_{1}+t_{2}\right]$, we have $\operatorname{card}\left[0, t_{1}+t_{2}\right] \leqslant \operatorname{card}\left[0, t_{1}\right] \operatorname{card}\left[t_{1}, t_{1}+t_{2}\right] \leqslant \alpha$. Thus $f_{3}\left[0, t_{1}+t_{2}\right]=\alpha$.

Let $\mathfrak{A}_{3}=\left\{f_{3}[a, b]:[a, b] \subset G, a<b\right\}$. From 3.1 and 1.6 we obtain:

3.2. THeorem. Let $G$ be an l-group, $a \epsilon \mathcal{A}_{3}, \alpha>s_{0}\left(\alpha=s_{0}\right)$. To any $g \in G$ there exists a greatest convex sublattice $B_{a}^{3}(g)$ of $G$ containing $g$ such that each non-trivial interval of $B_{a}^{3}(g)$ has the power a (the power $\left.\leqslant \mathrm{s}_{0}\right)$. The set $B_{a}^{3}(0)$ is an $l$-ideal of $G$ and $B_{a}^{3}(g)=B_{a}^{3}(0)+g$.

3.3. Let $G$ be a complete l-group. Then there exists a decomposition $G=A \times B$ such that (i) $A$ is a complete subdirect product of linearly ordered groups isomorphic to $N$, and (ii) $B$ does not contain any prime interval.

Proof. Let $f$ be an increasing cardinal property satisfying $\left(c_{1}\right)$ and $\left(c_{2}\right)$ (for example, $f=f_{1}$ ). Consider the complete subdirect decomposition with factors $C_{k}(k \in K)$ treated in 1.22 and 1.23. Let $K_{0}$ be the system of all $C_{k}$ isomorphic to $N$. We denote by $A(B)$ the set of all $g \in G$ such that $g_{k}=0$ for each $k \in K \backslash K_{0}\left(k \in K_{0}\right)$. Then clearly $G=A \times B$ and $A(B)$ is isomorphic to a complete subdirect prcduct of $l$-groups $C_{k}, k \in K_{0}$ $\left(k \in K \backslash K_{0}\right)$. Let $\left[t_{1}, t_{2}\right]$ be a non-trivial interval of $B$. Then $\left[t_{1}, t_{2}\right]$ is isomorphic to $[0, t], t \in B, t_{2}-t_{1}=t>0$, whence $t=\bigvee t_{k}\left(k \in K \backslash K_{0}\right)$. There exists $k_{1} \in K \backslash K_{0}$ such that $t_{k_{1}}>0$ and, since $C_{k_{1}}$ does not contain any prime interval, we have $t^{\prime} \in C_{k_{1}^{\prime}}, 0<t^{\prime}<t_{k_{1}}$. Therefore the intervals $[0, t]$ and $\left[t_{1}, t_{2}\right]$ are not prime.

3.4. Let $G$ be a complete $i$-group, $a \in G, a>0$, and assume that any disjoint subset of $G$ is finite. Then the lattice $[0, a]$ is isomorphic to a direct prodsct of a finite number of chains.

Proof. At first we shall prove that for each $b \in[0, a], b>0$ there is an element $b_{1}, 0<b_{1} \leqslant b$, such that $\left[0, b_{1}\right]$ is a chain. For otherwise there would exist $b_{1}, b_{2} \in[0, b], b_{1}>0, b_{2}>0, b_{1} \delta b_{2}$. Further, there would exist positive disjoint elements $b_{21}, b_{22} \in\left[0, b_{2}\right]$. In this way we could construct an infinite disjoint subset $\left\{b_{1}, b_{21}, b_{221}, \ldots\right\} \subset[0, a]$, which is a contradiction. Hence there exists a maximal disjoint subset $B$ $=\left\{b_{1}, b_{2}, \ldots, b_{n}\right\}$ of $[0, a]$ such that each interval $\left[0, b_{i}\right]$ is a chain. Since $G$ is Archimedean, for each $b_{i}$ there exists an integer $n_{i} \geqslant 1$ such that 
$n_{i} b_{i} \nless a$. Put $a_{i}=a \wedge n_{i} b_{i}$. The interval $\left[0, n b_{i}\right]$ is a chain for any integer $n$

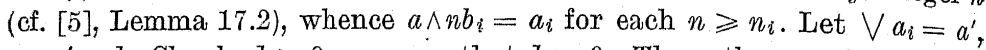
$a-a^{\prime}=k$. Clearly $k \geqslant 0$; assume that $k>0$. Then there exists $b_{i_{0}} \in B$ such that $k_{i_{0}}=k \wedge b_{i_{0}}>0$. Thus $a_{i_{0}}+k_{i_{0}} \leqslant\left(n_{i_{0}}+1\right) b_{i_{0}}, a_{i_{0}}+k_{i_{0}} \leqslant a^{\prime}+k=a$, whence $a_{i_{0}}+k_{i_{0}} \leqslant a \wedge\left(n_{i_{0}}+1\right), b_{i_{0}}=a_{i_{0}}$, a contradiction. Therefore $k=0$ and $a=\bigvee a_{i}$. Each interval $\left[0, a_{i}\right] \subset\left[0, n_{i} b_{i}\right]$ is a chain and the mapping $x \rightarrow\left\{x \wedge a_{i}\right\}(i=1, \ldots, n)$ is an ísomorphism of the lattice $[0, a]$ onto the direct product $\Pi\left[0, a_{i}\right](i=1,2, \ldots, n)$.

3.5. Let $G$ be a complete l-group and let $B$. have the same meaning as in 3.3. Let $[0, b] \subset B$ be a non-trivial $f_{3}$-homogeneous interval, $f_{3}[0, b]=a$. Then $a^{\aleph_{0}}=\alpha$.

Proof. At first assume that each disjoint subset of $[0, b]$ is finite. Then by 3.4 there exist elements $b_{1}, \ldots, b_{n} \in[0, b], b_{i}>0$, such that each interval $\left[0, b_{i}\right]$ is a chain and $[0, b]$ is isomorphic to the direct product of intervals $\left[0, b_{i}\right]$. According to $\left[\breve{s}\right.$, Thm. $\left.1^{\prime}\right]$ there exist $l$-ideals $B_{i}$ of $G$ such that $B_{i}$ are linearly ordered and $b_{i} \in B_{i}$. Moreover, $B_{i}$ are complete and since $B_{i} \subset B$ does not contain any prime interval, each $B_{i}$ is isomorphic to the additive $l$-group $R_{0}$ of all reals (cf. [1]); thus card $\left[0, b_{i}\right]=c$ and card $[0, b]=c=c^{N_{0}}$. Now let us suppose that there exists an infinite disjoint subset of the interval $[0, b]$; then there exists a disjoint subset $\left\{b_{1}, b_{2}, \ldots\right\}$ of $[0, b]$. Since any non-trivial interval of $B$ is infinite, card $[0, b]=\alpha \geqslant s_{0}$ and, according to the $\hat{j}_{3}$-homogenity of $[0, b]$, card $\left[0, b_{i}\right]=\alpha$ for $i=1,2, \ldots$ Write $b^{\prime}=\bigvee b_{i}(i=1,2, \ldots)$ and consider the mapping $\varphi: x \rightarrow\left\{x \wedge b_{i}\right\}$ of the lattice $\left[0, b^{\prime}\right]$ into $\Pi^{*}\left[0, b_{i}\right]$. By using the infinite distributivity of $\left[0, b^{\prime}\right]$ it is easy to verify that $\varphi$ is an isomorphism. Hence $\alpha=\operatorname{card}\left[0, b^{\prime}\right]=\operatorname{card} \Pi^{*}\left[0, b_{i}\right]=\alpha^{\boldsymbol{N}_{0}}$.

3.6. Let $G$ be a complete l-group and let $B$ be as $i_{i n}$ 3.3. Then $f_{3}$ satisfies $\left(c_{2}\right)$ with regard to $B$.

Proof. Let $t_{i} \in B, f_{3}\left[0, t_{i}\right]=\alpha(i=1,2, \ldots), 0<t_{1} \leqslant t_{2} \leqslant \ldots, \vee t_{i}=t$ and assume that all intervals $\left[0, t_{i}\right]$ are $f_{3}$-homogeneous. Since $B$ does not contain prime intervals, card $\left[0, t_{i}\right]=\alpha$ for $i=1,2, \ldots$ For any $x \in[0, t]$ we have $x=V\left(x \wedge t_{i}\right)$, whence the mapping $x \rightarrow\left\{x \wedge t_{i}\right\} \quad(i=1,2, \ldots)$ is a monomorphism of the set $[0, t]$ into the complete direct product $\Pi^{*}\left[0, t_{i}\right]$; from this we obtain $f_{3}[0, t]=\operatorname{card}[0, t] \leqslant \operatorname{card} \Pi^{*}\left[0, t_{i}\right]=\alpha^{N_{0}}$

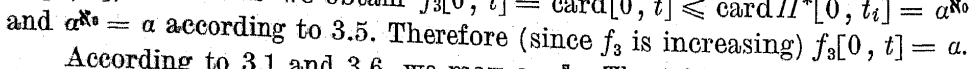
According to 3.1 and 3.6 , we may apply Th. 1.21 to the $l$-group $B$;
since $A$ is isomorphic to a complete subdirect product of linearly ordered
groups $C_{k}\left(k \in K_{0}\right)$ such groups $C_{k}\left(k \in K_{0}\right)$ such that any interval of $C_{k}$ is finite, we have the
following result:

3.7. THEOREM. Let $G$ be a complete l-group. Then $G$ is isomorphic to a complete subdirect product of $l$-groups $C_{k}(k \in K)$ such that, for each $C_{k}$,
che of the following conditions holds: (i) ang interoas che of the following conditions holds: (i) any interval of $C_{k}$ is finite and $C_{k}$ is linearly ordered, or (ii) any non-trivial interval of $C_{k}$ has the same cardinality $\alpha_{k}$ and $\alpha_{k}^{k_{0}}=\alpha_{k}$.

Let $\alpha$ be a cardinal, $\alpha^{\aleph_{0}}=\alpha$. Then there is a lattice ordered group $G_{a} \neq\{0\}$ such that card $[a, b]=\alpha$ for each non-trivial interval of $G_{a}$. We construct $G_{\alpha}$ as follows:

Since $\alpha \boldsymbol{\aleph}_{0}=\alpha$, there exists a Boolean algebra $B_{\alpha} \neq\{0\}$ such that card $\left[b_{1}, b_{2}\right]=\alpha$ for any non-trivial interval of $B_{\alpha}$ (cf. Pierce [6]). Let $E$ be the vector lattice consisting of all elementary Carathéodory functions on $B_{a}$ (cf. Goffman [3]); i.e., $E$ is the set consisting of all forms

$$
f=a_{1} b_{1}+\ldots+a_{n} b_{n}
$$

(where $a_{i} \neq 0$ are reals and $b_{i} \in B_{\alpha}, b_{i}>0, b_{i_{1}} \wedge b_{i_{2}}=0$ for any $i_{1}, i_{2}$ $\epsilon\{1, \ldots, n\}, i_{1} \neq i_{2}$ ) and of the "empty form"; if $g$ is another such form,

$$
g=a_{1} b_{1}^{\prime}+\ldots+a_{m}^{\prime} b_{m}^{\prime}
$$

then $f, g$ are considered as equal if $\bigvee_{i=1}^{n} b_{i}=\bigvee_{j=1}^{m} b_{j}^{\prime}$ and if $a_{i}=a_{j}^{\prime}$ whenever $b_{i} \wedge b_{j}^{\prime} \neq 0$. For any $b, b^{\prime} \in B_{\alpha}$ let $b-b^{\prime}$ be the relative complement of $b \wedge b^{\prime}$ in the interval $[0, b]$. The operation + in $E$ is defined by

$$
f+g=\sum_{i=1}^{n} \sum_{j=1}^{m}\left(a_{i}+a_{j}^{\prime}\right)\left(b_{i} \wedge b_{j}^{\prime}\right)+\sum_{i=1}^{n} a_{i}\left(b_{i}-\bigvee_{j=1}^{m} b_{j}^{\prime}\right)+\sum_{j=1}^{m} a_{j}^{\prime}\left(b_{j}^{\prime}-\bigvee_{i=1}^{n} b_{i}\right),
$$

where in the summations only those terms are taken into account in which $a_{i}+a_{j}^{\prime} \neq 0$ and the elements $b_{i} \wedge b_{j}^{\prime}, b_{i}-\bigvee_{j=1}^{m} b_{j}^{\prime}, b_{j}^{\prime}-\bigvee_{i=1}^{n} b_{i}$ are non-zero. The multiplication by a real $a \neq 0$ is defined by $a f=\left(a a_{1}\right) b_{1}+\ldots+\left(a a_{n}\right) b_{n}$; of is the empty form. The form (2) is positive, if $a_{i}>0$ for $i=1, \ldots, n$. Let $G_{\alpha}$ be the subset of $E$ consisting of the empty form $f_{0}$ and of all forms (2) such that $a_{i} \neq 0$ are integers $(i=1,2, \ldots, n)$. Then $G_{a}$ is an $l$-subgroup of the $l$-group $E$ and card $G_{a}=\alpha$. For proving that card $[f, g]=\alpha$ for any non-trivial interval $[f, g]$ of $G_{\alpha}$ it suffices to examine the intervals $\left[f_{0}, f\right], f>f_{0}$. Let $f \in G_{a}$ be the form (2) with $a_{i} \geqslant 1(i=1, \ldots, n)$. Let

$$
Y=\left\{b \in B_{a}: 0<b \leqslant b_{1}\right\}, \quad \bar{Y}=\left\{g \in G_{a}: g=1 b, b \in Y\right\} .
$$

Since $\operatorname{card}\left[0, b_{1}\right]=\alpha$, we have card $\bar{Y}=\alpha$ and, because $\bar{Y} \subset\left[f_{0}, f\right] \subset G_{a}$, $\operatorname{card}\left[f_{0}, f\right]=\alpha$.

It remains as an open question whether for any cardinal $\alpha$ satisfying $\alpha \aleph_{0}=\alpha$ there exists a complete $l$-group $G$ such that $\operatorname{card} L=\alpha$ for any non-trivial interval of $G$.

Analogously as in $\S 2$ we may define the reduced power card ${ }^{*} L$ of a bounded lattice $L$ to be the least cardinal $\alpha$ such that there exists a dense subset $L_{1}$ of $L, \operatorname{card} L_{1}=\alpha$. Write $f_{4} L=\max \left\{\operatorname{card}^{*} L, s_{0}\right\}$. Obviously $f_{4}$ is 
increasing, but $f_{4}$ fails to satisfy the condition $\left(c_{1}\right)$. Example: Let $G=R_{0} \times R_{0}, \quad g_{0}=(0,0), \quad g_{1}=(1,0), \quad g_{2}=(0,1) . \quad$ Clearly, $\quad f_{4}\left[g_{0}, g_{1}\right]$ $=f_{4}\left[g_{0}, g_{2}\right]=s_{0}$ and the intervals $\left[g_{0}, g_{1}\right],\left[g_{0}, g_{2}\right]$ are $f_{4}$-homogeneous. Let $L_{1}$ be a dense subset of $\left[g_{0}, g\right], g=g_{1}+g_{2}=(1,1)$. Let $r \in[0,1]$, $h_{1}=(0, r), h_{2}=(1, r)$. Then there exists $g_{r} \in L_{1} \cap\left[g_{1}, g_{2}\right]$ and $g_{r}=\left(x_{r}, r\right)$, $x_{r} \in[0,1]$. Thus $g_{r_{1}} \neq g_{r_{2}}$ whenever $r_{1} \neq r_{2}$ and therefore card $L_{1}=c$ $=f_{4}\left[0, g_{1}+g_{2}\right] \neq f_{4}\left[0, g_{1}\right]$.

\section{References}

[1] G. Birkhoff, Lattice theory, Amer. Math. Soc. Colloquium Publications, Vol. 25, Third Edition, 1967.

[2] L. Fuchs, Частично упорядоченные алгебраические системы, Москва 1965.

[3] C. Goffman, Remarks on lattice ordered groups and vector lattices. I. Carathéodory functions, Trans. Amer. Math. Soc. 88 (1958), pp. 107-120.

[4] J. Jakubík, Remark on the Jordan-Dedekind condition for Boolean algebras, Casopis pěst. mat. 82 (1957), pp. 44-46.

[5] J. Jakubik, Konvexe Ketten in l-Gruppen, Časopis pěst. mat. 84 (1959); pp. 53-63.

[6] R. S. Pierce, A note on complete Boolean algebras, Proc. Amer. Math. Soc. 9 (1958), pp. $892-896$

[7] R. S. Pierce, Some questions about complete Boolean algebras, Proceedings of Symposia in pure matematics, Vol. II, Lattice Theory, Amer. Math. Soc., 1961.

[8] B. Z. Vulich, Введение в теорию полуупорлдоченных пространств, Москва 1961.

Regu par la Rédaction le 28. 4. 1969

\section{Algèbre du calcul propositionnel trivalent de Heyting}

par

\section{Luiz Monteiro (Bahia Blanca)}

1. Introduction. Nous nous proposons dans cette note de déterminer le nombre d'éléments de l'algèbre $H_{3}$ avec un nombre fini de générateurs libres $\left.{ }^{1}\right)$.

1.1. DÉfintrion. Une algèbre de Heyting $\left({ }^{2}\right) A$ sera dite une algèbre $H_{3}$ si l'égalité suivante est vérifiée:

$$
((a \rightarrow c) \rightarrow b) \rightarrow(((b \rightarrow a) \rightarrow b) \rightarrow b)=1
$$

quels que soient les éléments $a, b$ et $c$ de $A$.

Ces algèbres jouent dans l'étude du calcul propositionnel trivalent de Heyting (A. Heyting [5], J. Łukasiewicz [6], I. Thomas [16]) un rôle analogue à celui des algèbres de Boole dans le calcul propositionnel classique.

Il est évident que toute algèbre de Boole, est une algèbre $H_{3}$, car dans les algèbres de Boole est valable l'égalité $(b \rightarrow a) \rightarrow b=b$, qui implique ( $\mathrm{T}$ ).

Indiquons l'exemple le plus simple d'une algèbre $H_{3}$, qui n'est pas une algèbre de Boole: Soit $T=\{0, a, 1\}$ l'ensemble formé par trois éléments distincts sur lequel on définit les opérations $\Lambda, \vee$ et $\rightarrow$ au moyen des tables suivantes (auxquelles nous ajoutons la table de l'opération de négation 7 définie par $\neg x=x \rightarrow 0$ ).

\begin{tabular}{|c|c|c|c|c|c|c|}
\hline$\wedge$ & $\mid \begin{array}{lll}0 & a & 1\end{array}$ & V & $\begin{array}{lll}0 & a & 1\end{array}$ & $\rightarrow$ & $\left|\begin{array}{lll}0 & a & 1\end{array}\right|$ & $7 x$ \\
\hline 0 & $\begin{array}{lll}0 & 0 & 0\end{array}$ & 0 & $0 a 1$ & 0 & $\begin{array}{lll}1 & 1 & 1\end{array}$ & 1 \\
\hline$a$ & $\begin{array}{lll}0 & a & a\end{array}$ & $a$ & $\begin{array}{lll}a & a & 1\end{array}$ & $a$ & $\begin{array}{lll}0 & 1 & 1\end{array}$ & 0 \\
\hline 1 & $\begin{array}{lll}0 & a & 1\end{array}$ & 1 & 111 & 1 & $\left|\begin{array}{lll}0 & a & 1\end{array}\right|$ & 10 \\
\hline
\end{tabular}

Cette algèbre a été considérée pour la première fois par A. Heyting (1930)

L'algèbre de Boole $B=\{0,1\}$ est une sous-algèbre de $T$ que nous aurons à utiliser par la suite.

(1) Un résumé de cette note a été publié dans Notas de Lógica Matemática № 19 (1964). $\left({ }^{2}\right)$ Voir: T. Skolem [14], G. Birkhoff [1], p. 459, [3], p. 147, M. Ward [17] et
A. Monteiro [8]. Nous avons adopté la terminologie de H. Rasiowa et R. Sikorski [11]. 